(Aus der Poliklinik des Herrn Prof. H. Oppenherm in Berlin.)

\title{
Ueber eine typische Verlaufsform der multiplen Sklerose.
}

\author{
Von \\ Dr. D. Frank.
}

Die Symptome, welche das Krankheitsbild der multiplen Sklerose einleiten, können der Natur des Leidens entsprechend äusserst mannigfaltig sein. Doch sind es eine Anzahl Erscheinungen, welche mit einer gewissen Regelmässigkeit wiederkehren und auf diese Weise den Beginn und die Verlaufsart der Krankheit bestimmen. So bilden in einer Anzahl von Fällen spastische Erscheinungen und motorische Schwäche der Beine das erste Symptom der Krankheit, in anderen Fällen sind es cerebrale Symptome, welche den Grund der ersten Klagen des Patienten bilden u. s. w. Wenig Beachtung in ihrer Eigenschaft als Initialsymptom der multiplen Sklerose haben die Störungen von Seiten des Sehvermögens erfahren, trotz der eingehenden Behandlung, die gerade diesen Störungen zu Theil geworden ist. Zwar finden wir eine Anzahl von Beobachtungen dieser Art in der Literatur verzeichnet, ohne dass aber die Beobachter diese Thatsache weiter berücksichtigt hätten. Auch diejenigen Autoren, welche sich speciell mit der Erforschung der Sehstörungen bei der multiplen Sklerose beschäftigt haben, erwähnen das gelegentliche Auftreten von Opticusaffectionen als erstes Symptom dieser Krankheit, als einer interessanten Erscheinung, gehen aber nicht weiter auf die Thatsache selbst ein oder betrachten sie als seltenes Vorkommniss. So hebt (Gnauck ${ }^{1}$ ) als besonders bemerkenswerth hervor, dass in einem seiner Fälle die Krankheit mit einer Sehstörung begann, welche es ihm ermöglichte, trotz der Geringfígigkeit der sich später zugesellenden Symptome die Diagnose auf beginnende multiple Sklerose zu stellen. Uhthoff2) spricht von dem Beginn der multiplen Sklerose mit einer uncomplicirten Sehstörung, die lange Zeit den anderen Symptomen vorausgehen kann, wie von einer interessanten Erscheinung, hält sie aber für recht selten. Ebenso scheint Lübbers ${ }^{3}$ ) ein solches Vorkommen eine Seltenheit zu sein.

1) Berliner klinische Wochenschrift 1889. Nr. 27.

2) Untersuchungen über die bei der multiplen Sklerose vorkommenden Augenveränderungen. Berlin 1889. Th. II.

3) Augenveränderungen bei disseminirter Sklerose. Archiv f. Psychiatrie. 1897. XXIX, 3. 
Oppenheim 1), welcher als einer der ersten auf die grosse Rolle, welche Opticusaffectionen im klinischen Bilde der multiplen Sklerose spielen, wo sie oft neben spastischer Parese die einzigen klinisch zu Tage tretenden Symptome bilden, aufmerksam macht, betont in einer neueren Arbeit²), dass das Leiden mit einer Sehstörung einsetzen kann, und im Gegensatz zu den anderen Autoren hält er diese Art des Beginns für eine häufige Erscheinung. ${ }^{3}$ ) In der Poliklinik des Herrn Prof. Oppenheim wurde eine Anzahl von Fällen beobachtet, welche nicht nur eine solche Meinung rcchtfertigen, sondern welche es auch gestatten, von einer Verlaufsform der multiplen Sklerose zu sprechen, für die der Beginn mit einer den anderen Symptomen zuweilen jahrelang vorausgehenden Sehstörung geradezu typisch ist. In Folge dessen wurde ich von Herrn Prof. Oppen heim aufgefordert, diese Fälle zu sammeln und der Oeffentlichkeit zu übergeben.

Fall 1. J. F., Stenograph, 24 Jahre alt, kam in die Poliklinik am 25. März dieses Jahres. Patient stammt ans gesunder Familie und will bis za seinem 17. Lebensjahr gesund gewesen sein. Um diese Zeit hatte er mehrere Wochen angestrengt bei Licht gearbeitet und bekam eine Sehnervenentzündung auf dem linken Auge. Das rechte Auge war gar nicht betroffen. Die Krankheit nahm schnell zu bis zu dem Grade, dass Patient mit dem linken Auge nur diffuses Licht unterscheiden konnte, und nachdem sie etwa sieben Wochen gedauert, begann sie sich langsam zu bessern, ohne jedoch vollständig in Heilung überzugehen. Das linke Auge blieb bedeutend geschwächt. Seit jener Zeit war Patient im Uebrigen vollständig gesund, hat nur einmal eine leichte Influenza durchgemacht, die ohne ärztliche Behandlung ausheilte. Vor einem Jahr begann Pat. Schwäche in den Beinen zu spüren, die immer mehr und mehr zunahm und sich besonders in der letzten Zeit verstärkte.

Status praesens. Kräftig gebanter, gut genährter Mann. Lues und Potus negirt. Von Seiten der inneren Organe keine bemerkenswerthen Störungen. Patient klagt über Schwäche der Beine. Keine Harnbeschwerden. Deutlicher Nystagmus und Beweglichkeitsbeschränkung beider Bulbi nảch beiden Seiten. Linker Opticus in toto erheblich blässer als normal, namentlich die temporale Papillenhälfte. Sehschärfe auf demselben stark herabgesetzt - auch grosse Schrift wird nicht gelesen; Fin Entfernung gezählt.

Motorische Schwäche in beiden Beinen, Kniephänomen sehr stark, in der Rückenlage deutliche Zeichen von Rigidität: Fussclonus, Patellarclonus deutlich. Beim Kniehackenversuch macht sich ein Wackeln bemerkbar, das bei Augenschluss nicht zunimmt. Auch bei Beugung der Beine bisweilen ein gröberes Wackeln. Der Gang ist nicht dentlich spastisch, sondern vor Allem durch cerebellare Incoordination schwankend, unsicher; beim Kehrtmachen kommt Patient ins Taumeln.

1) Zur Pathologie der disseminirten Sklerose. Berl. kl. Wochenschrift 1887. 48.

2) Zur Lehre von der multiplen Sklerose. Berl. klin. Wochenschr. 1896. Nr. 9.

3) Lehrbuch der Nervenkrankheiten. II. Aufl. Berlin 1898. S. 25\%. 
Dieser Fall kennzeichnet sich durch die Entwicklung des Krankheitsprocesses in zwei, durch einen Zeitraum von sechs Jahren getrennten Phasen. Die erste enthält die einseitige Sehstörung, welche unter dem Einfluss von Ueberanstrengung der Augen in acuter Weise entstand und in kurzer Zeit ihren Höhepunkt erreicht, um dann nach theilweisem Zurückgehen der Erscheinung stationär zu bleiben. Die zweite umfasst die lange Zeit nachher allmählich sich entwickelnde spastische Parese und cerebellare Ataxie. Durch seine Entwicklung in zwei. Perioden, sowie durch den Zeitraum, der das Auftreten der Sehstörung von dem der anderen Symptome trennt, erinnert dieser Fall an einen von Eulenburg ${ }^{1}$ ) beschriebenen.

Fall 2. ${ }^{2}$ ) E., Tischler, 31 Jahre alt, stammt angeblich aus gesunder Familie; Lues und Potus werden verneint. Die Frau des Patienten abortirte einmal mit drei Monaten. Patient will bis zum Jahre 1893 vollständig gesund gewesen sein. Um diese Zeit bemerkte er plötzlich, dass er anfing schlecht zu sehen. Die Sehschwäche nahm schnell zu und erreichte nach einem Monat einen solchen Grad, dass Patient keine Gegenstände mehr unterscheiden konnte. Auch die Farbenempfindung hatte gelitten, Roth und Grün erkannte Patient gar nicht, die Empfindung für Weiss war beeinträchtigt, nur Blau unterschied er gnt. Patient wurde in der Schweig ger'schen Klinik behandelt und nach drei Wochen trat eine Besserung ein, die aber nicht lange anhielt; das Sehvermögen wurde wieder schlechter und kam allmählich in den Zustand, in dem es sich jetzt befindet, ohne aber den Grad von Schwäche zu erreichen, den es während der ersten Attacke gehabt hatte. Nach einem Jahre stellten sich Schwindelanfälle ein, welche nicht mit Kopfschmerz und Erbrechen einhergingen. Später das Gefühl von Spannung in den Beinen. Patient befindet sich in Behandlnng der Poliklinik seit dem 23. September 1895. Er kam damals mit Klagen über Gefühl von Spannung um den Leib herum und in den Beinen, sowie über Erschwerung des Urinlassens. Objectiv wurde festgestellt: Parese des linken Abducens, Nystagmus, beiderseits weisse Verfärbung der Papillae nervi optici bis fast zum Rande; keine motorische Schwäche in den Armen und Beinen, keine Erhöhung der Sehnenphänomene und keine Spasmen. Im Laufe der nächsten Zeit hörten die Urinbeschwerden auf, auch das Spannungsgefühl um den Leib liess zeitweilig nach, dagegen stellten sich Schwäche und Steifigkeit der Beine ein, welche allmählich immer mehr nnd mehr zunahmen. Gegenwärtig bietet Patient folgendes Bild:

Hochgewachsener, kräftig gebauter Mann von sehr schlechtem Ernährungszustand. Haut und Schleimbäute sind sehr blass, Fettpolster fehlt fast vollständig. An den unteren Extremitäten zahlreiche Narben. Die Augenbewegungen sind frei, in den Endstellungen nystagmusartige Zuckungen, Pupillenraction prompt. Weisse Verfärbung beider Papillen; der Patient zählt Finger bis auf 2-3 Meter Entfernung, auch grobe Schrift wird nicht

1) Neurologisches Centralblatt. 1884. Nr. 22.

2) Dieser Fall, der auch in anderer Hinsicht sehr interessant ist, ist bereits ron Herrn Dr. Cassirer beschrieben worden. Deutsche med. Wochenschr. 1896. Nr. 43. 
erkannt. Achromatopsie für Roth und Grün. Die Zunge wird gerade herausgestreckt, zittert leicht fibrillär. Die Sprache ist nicht merklich verändert. Im linken Arm bedeutende motorische Schwäche, die Sehnenphänomene an den Armen erhöht, auch merkliche Rigidität derselben. Patellarreflexe stark gesteigert, Fussclonus, Rigidität der Beine sehr deutlich, motorische Kraft im linken Bein gering. Bauch - und Cremasterreflex deutlich. Kein Romberg, keine Ataxie; Gang ausgesprochen spastisch - das linke Bein wird nachgeschleppt. Keine Beschwerden beim Urinlassen, Intentionszittern nicht nachzuweisen. Patient lacht fast beständig, behauptet das Lachen nicht zurückhalten zu können.

Dieser Fall zeigt ebenso wie der folgende das Typische der uns beschäftigenden Verlaufsform besonders. deutlich. Bei einem bis dahin gesunden, weder an Lues leidenden, noch unter dem Einfluss einer Intoxication stehenden Manne entwickelt sich eine Affection des $\mathrm{N}$. opticus, welche nach kurzer Zeit zur Atrophie desselben führt, die aber nur eine unvollständige bleibt. Erst nach einem Jahre beginnen sich andere Symptome der Krankheit zu entwickeln, als deren Vorläufer die ätiologisch nicht begründete Opticusaffection aufgetreten war. Ein solches Verhalten ist diagnostisch von grosser Wichtigkeit, indem es darauf hinweist, dass in Fällen von plötzlich auftretenden Sehstörungen, für welche sonst keine Begründung sich finden lässt, die Möglichkeit einer späteren Entwicklung der multiplen Sklerose im Auge behalten werden muss.

Fall 3. C. B., Stellmacher, 27 Jahre alt, kam am 20. Mai dieses Jahres in die Poliklinik mit Klagen über Schwäche in den Beinen, die seit sechs Wochen bestehe, brennendem Schmerz in der linken Bauchgegend, Beschwerden beim Urinlassen und über zunehmende Gedankenschwäche. Auch die Sprache soll seit einiger Zeit langsamer geworden sein; ebenso behauptet Patient häufig ohne Grund lachen zu müssen, obwohl „ihm gar nicht darnach sei". Die Angehörigen des Patienten sollen alle gesunde Leute sein - ein Bruder ist blind; er selbst will bis zur letzten Zeit vollständig gesnnd gewesen sein, erst auf eindringliches Befragen giebt er zll, dass er vor drei Jahren kurze Zeit an "Papillitis" gelitten habe, wegen welcher er in der Charité behandelt wurde (Schwitzcur) und die vollständig vergangen sei. Jetzt sei das Sehen gut, nur bei längerer Anstrengung würde ihm etwas dunkler vor den Augen.

Status praesens. Mittelgrosser, schwächlich gebauter Mann. Starke Abmagerung, Haut und Schleimhäute sind sehr blass. Die Augenbewegungen sind frei - in den seitlichen Endstellungen nystagmusartige Zuckungen. Die rechte Papille ist etwas abgeblasst, die Pupillenreaction normal. Das Kniephänomen ist beiderseits erheblich gesteigert; - Rigidität der Beine, ausgesprochener Fussclonus. Keine erhebliche motorische Schwäche in den Beinen. Die Sensibilität ist überall normal. Schwanken bei Augenschluss. Der Gang ist atactisch, anch ein wenig klebend. Anch in der Ruhelage deutliche Ataxie. Die Sprache ist etwas verlangsamt, aber kein Silbenstolpern, kein Scandiren. Zwangslachen. Patient schildert typisches Inten- 
tionszittern,- welches aber gegenwärtig nicht nachzuweisen ist. Von Seiten der inneren Organe nichts Bemerkenswerthes.

Hier zeichnet sich die Sehstörung, welche drei Jahre vor dem Ausbruch der anderen Erscheinungen der Krankheit auftrat, durch ihren flüchtigen Verlauf aus, indem sie in kurzer Zeit, unter Zurücklassung nur geringfügiger Spuren, ausheilte. In Folge dieses Verhaltens hielt Patient die Thatsache gar nicht für erwähnenswerth und musste erst durch wiederholtes Befragen an dieselbe erinnert werden.

Fall 4. Frau K., befindet sich in Behandlung der Poliklinik seit dem Mai 1895. Patientin stammt aus einer Familie, in welcher Tuberculose häufig ist; hat einmal abortirt, ein Kind starb 14 Tage nach der Geburt, ein zweites lebt und soll gesund sein. Im Jahre 1891 hatte sich 14 Tage nach einer schweren Entbindung Sehschwäche auf dem linken Auge eingestellt, zu der sich nach kurzer Zeit Doppeltsehen hinzugesellte; beide Störungen vergingen sehr bald. Im Juni 1892, ein paar Wochen nach einem zweiten Wochenbett bemerkte Patientin zunächst Kälte und Schwäche im rechten Bein, bald darauf auch im rechten Arm. Innerhalb des ersten Monates verschlimmerte sich der Zustand bedeutend, besserte sich dann allmählich, ohne aber vollständig zu verschwinden. Auch die Sehstörung auf dem linken Auge, welche sich von Nenem eingestellt hatte, besserte sich von selbst. Die Beschwerden im rechten Bein bildeten auch den Grund der Klagen, wegen welcher Pat. zum ersten Mal die Poliklinik aufsuchte. Damals wurde constatirt: Abblassung der linken Papille in toto und $\mathrm{Ab}$ schwächung der Sehschärfe auf dem linken Auge. Deutliche Rigidität der Beine und erhebliche motorische Schwäche, ausserdem im rechten Wackeltremor. Breitbeiniger, spastischer Gang, stark gesteigerte Reflexerregbarkeit an den Beinen. Neigung zum Lachen ohne jeglichen Grund und eigenthïmliche psychische Zustände von mehrere Stunden langer Dauer, in denen Pat. verstimmt, reizbar ist, nicht weiss, was sie thut, Neigung hat, Alles zu zerstören. -- Der geschilderte Zustand erfuhr in der nächsten Zeit geringe Aenderungen, indem bald Besserung, bald wieder Verschlimmerung eintrat. Im Laufe des letzten Jahres hatte eine Zeit lang Incontinentia urinae bestanden; vom September bis December vorigen Jahres war das rechte Auge blind, hat sich aber von selbst vollständig erholt. Gegenwärtig klagt Pat. hauptsächlich über Zittern in der linken Hand, welches sich vor Kurzem eingestellt hat; anch die Beine sind in der letzten Zeit besonders schlecht geworden.

Status praes. Hochgewachsene, ziemlich schlecht genährte Person. Der Blick ist starr. die Augenbewegnngen frei Kein Nystagmus. Beide Papillae nervii optici sind in toto etwas blasser als normal, rechte kanm als pathologisch zu deuten, links auch nur als geringgradige Atrophie, Herabsetzung der centralen Sehschärfe ist gegenwärtig nicht nachzuweisen; auch eine gröbere Herabsetzung des excentrischen Sehens (durch Handbewegungen geprüft) ist nicht vorhanden. In der linken Hand typisches Intentionszittern. Motorische Schwäche und Rigidität beider Beine, im linken etwas stärker ausgesprochen. Patellarclonus, Fussclonus. Das Gehen ist äusserst erschwert, Pat. schleppt sich, sich an Gegenstände anklammernd, die Füsse 
am Boden schleifend, mühsam fort. Die Sprache ist nicht scandirend, das Gedächtniss bedeutend beeinträchtigt.

Fall 5. Fran E. D., Hausverwaltersfrau, 49 Jahre alt, stammt aus gesunder Familie, hat vier gesunde Kinder. Pat. giebt an etwa vor sechs Jahren die ersten Zeichen ihres jetzigen Leidens bemerkt zu haben. Es stellte sich ein Taubheitsgefühl in den Fingern ein, etwas später dasselbe Gefühl in den Füssen. Einige Monate vorher war eine Sehschwäche aufgetreten, welche sich gleichzeitig auf beiden Augen entwickelt hatte und welche Anfangs so geringgradig war, dass Pat. gar nicht darauf achtete, bis ihre Umgebung sie darauf aufmerksam machte, dass sie auch nahe Gegenstände nicht erkenne. Sie consultirte dann einen Arzt, der aber „an den Augen nichts finden konnte". Nach einiger Zeit verlor sich die Sehschwäche vollständig. Circa zwei Jahre später stellte sich Kopfzittern ein, nachdem schon längere Zeit vorher Schwäche in den Beinen sich bemerkbar gemacht hatte. Um dieselbe Zeit bemerkte Pat. Verlangsamung der Sprache; zeitweise traten Schwindelanfälle auf. Auch die Sehstörung hatte sich wieder eingestellt. In die Poliklinik kam sie zum ersten Mal im September 1896. Es bestanden damals zeitweise Harnbeschwerden und Gedankenschwäche. Objectiv fanden sich typisches Kopfwackeln, Intentionszittern, scandirende Sprache, spastische Erscheinungen und motorische Schwäche beider Beine, Fussclonus. Der Augenhintergrund erwies sich im Ganzen als normal, die temporale Papillenhälfte vielleicht ein wenig blasser. - Diese Erscheinungen nahmen im Laufe der nächsten Jahre allmählich immer mehr und mehr zu. Gegenwärtig bietet Pat. folgendes Bild: Vollständige Paraplegie beider Beine. Pat. ist nicht im Stande sich ohne Unterstiutzung auf den Beinen zu halten. Starkes Wackeln des Kopfes, wenn er aus der Ruhelage erhoben wird. Die Augenbewegungen sind frei, in den Endstellungen deatlicher Nystagmus. Die Sehschärfe ist bedeutend herabgesetzt, nur gröbste Schrift wird gelesen, Finger werden auf $1 \frac{1 / 2}{}$ Meter Entfernung schlecht gezählt. Typisches Intentionszittern in beiden Händen. Starke Rigidität beider Beine. Die Sehnenphänomene an beiden Beinen so sehr gesteigert, dass ein leiser Druck genügt, sie auszulösen. Keine Harnbeschwerden gegenwärtig. Pat. weint und lacht durcheinander ohne jeglichen Grund.

In den letzten beiden Fällen trat die Sehstörung unmittelbar vor dem Ausbruch der anderen Erscheinungen auf. In dem ersten Falle waren es Störungen von Seiten der Augenmuskeln, welche sich an die Sehstörung anschlossen, im zweiten Falle Parästhesien in den Händen und Füssen. Immerhin bleibt der Beginn mit einer Affection der Nervus opticus bemerkenswerth.

Fall 6. E. S., 23 Jahre alt, verheirathet, Arbeiterin in einer Hutfabrik. Der Vater der Patientin starb an Lungentuberculose. Patientin kam in die Poliklinik am 29. Juli dieses Jahres mit Klagen über häufigen Kopfschmerz mit Uebelkeit, Gefühl von Schwindel, Schwäche und Steifigkeit in den Beinen und Abschwächung des Sehvermögens. Alle diese Erscheinungen wären in der letzten Zeit aufgetreten. Als Kind von 10 Jahren hat Patientin Diphtheritis durchgemacht, sonst war sie bis zur letzten Zeit gesund. Vor einem Jahre bemerkte sie, dass die Augen schwächer wurden; 
sie sah undeutlich, die Gegenstände erschienen oft verschwommen, hänfig "lag es wie ein Nebel vor den Augen", besonders nach den geringsten Anstrengungen. Diese Sehschwäche hat sich, ohne besonders zuzunehmen, bis in die letzte Zeit erhalten.

Status praesens. Mittelgrosse, leidlich gut genährte Person. Die inneren Organe sind vollständig gesund. Der Schädel ist nicht druckempfindlich. Die Augenbewegungen sind frei; kein Nystagmus. Die Pupillenreaction normal. Die Zunge wird gerade hervorgestreckt, zittert leicht fibrillär, der Mund ist etwas nach rechts verzogen, die Differenz bleibt bei mimischen Bewegungen nicht bestehen. Tricepsphänomen leicht erhöht, leichte Ataxie und Tremor in der rechten Hand beim Finger-Nasenversuch, dentliche A taxie und Wackeltremor der linken Hand, auch beim Greifen nach Gegenständen. Die motorische Kraft in den beinen ist gering. Deutliche, wenn auch nicht erhebliche Rigidität im linken Bein, stärkere im rechten; Patellarreflex rechts bedentend erhöht, im erhobenen rechten Bein beträchtlicher Wackeltremor. Rechts Andeutung von Fussclonus. Die Sensibilität ist überall intact. Die ophthalmoskopische Untersuchung ergiebt keine Veränderung der Papillen. Die Sprache ist etwas verlangsamt. Patientin giebt an, hänfig lachen zu müssen, auch ohne Grund.

Hier documentirt siçh die Sehstörung durch den Mangel an jeglicher ophthalmoskopisch nachweisbarer Veränderung. Trotzdem kann es nicht zweifelhalt sein, dass die Sehstörung anch in diesem Falle das erste Symptom der sich entwickelnden multiplen Sklerose bildete. Die bestimmten Angaben über die Sehstörung, die fortschreitende Entwicklung der anderen Symptome der Krankheit lassen bei dem Mangel anderer Zeichen eines functionellen Leidens den Gedanken an eine blos functionelle Störung des Sehvermögens nicht aufkommen.

Dem Krankenjournal entnehme ich noch folgende Angaben über drei hierhergehörige Fälle.

Fall 7. G. 0 ., 39 Jahre alt, kam in die Poliklinik mit Klagen über Schwäche und Steifigkeit in den Beinen. Von 9 Jahren Sehschwäche. Patient bemerkte, dass er anfing schlecht zu sehen. Die Sehschwäche nahm immer mehr zu. Mehrere Jahre darauf eine Zeit lang Doppeltsehen. Bei der Untersnchnng fanden sich die ausgesprochenen Zeichen der multiplen Sklerose. Ophthalmoskopisch erwies sich die rechte Papille in toto atrophisch verfärbt, besonders ausgesprochen in der temporalen Papillenhälfte.

Fall 8. Frl. M., erkrankte plötzlich an einer linksseitigen Neuritis optica. Gleichzeitig trat Schwäche in den Beinen auf. Nach einem Jahre spastische Parese der Beine, Wackeltremor in denselben, Zittern, Anfälle von Schwindel; die ophthalmoskopische Untersuchung ergab links eine Abblassung der temporalen Papillenhälfte.

Fall 9. E. A., 31 Jahre alt, klagt über zunehmende Schwäche in den Beinen. Seit einem halben Jahr hat die Sehkraft bedeutend abgenommen. Vor mehreren Monaten kurze Zeit Incontinentia urinae. Gegenwärtig beiderseits leichter Nystagmus, stark erhöhte Patellarreflexe, Fusszittern, verlangsamte Sprache. Ophtalmoskopisch: beiderseits temporal abgeblasste Papillenhälften. Die Sehschärfe ist rechts ${ }^{15}{ }_{70}$, links $15 / i_{1}$. Rechts grosses absolutes centrales Scotom für Grün, links relatives centrales Scotom. 
Auf Grund der angeführten Fälle sind wir wohl berechtigt, den Beginn der multiplen Sklerose mit einer Sehstörung als kein so seltenes Vorkommniss zu betrachten, sondern für eine Anzahl von Fällen einen solchen Beginn geradezu als typisch anzusehen. Der Grund für die bisherige Anschauung liegt in dem Umstande, dass die Störungen des Sehvermögens zuweilen Jahre lang den anderen Symptomen der Krankheit vorausgehen und dass sie besonders, wenn sie nur geringe Beschwerden verursachen, leicht aus dem Gedächtniss der Patienten verschwinden und vor den späteren, das subjective Empfinden stärker beeinträchtigenden Störungen zurücktreten. So gelang es, in mehreren unserer Fälle erst auf genaues und eindringliches Befragen die Thatsache des früheren Leidens festzustellen und zeigten sich die Patienten sehr erstaunt, dasselbe in Zusammenhang mit der gegenwärtigen Krankheit gebracht zu sehen. Unter 59 Fällen von multipler Sklerose, welche in der Poliklinik des Herrn Prof. Oppenheim zur Beobachtung kamen, zeigten 9, also mehr als 15 Proc., den Beginn des Leidens mit einer Sehstörung. In einer neulich von Probst ${ }^{1}$ ) gebrachten Zusammenstellung fanden sich unter 35 Kranken 4, also 11 Proc., mit gleichem Beginn der Krankheit. Eine so bäufige Wiederholung dieser Erscheinung benimmt aber derselben den Charakter des Zufälligen und weist vielmehr auf eine typische Art der Entwicklung dieses Leidens. Mit der Anerkennung dieser Thatsache erwächst die Aufgabe in allen Fällen von spastischer Parese der Beime nach einer früher etwa vorausgegangenen Sehstörung zu forschen. Viele ihrem Wesen nach unklare Krankheitsfälle würden sich auf diese Weise in das Bild der multiplen Sklerose einreihen lassen. So könnte z. B. in manchen Fällen, wo die Entscheidung, ob letztere oder Hirnsyphilis vorliegt, schwierig ist, die Feststellung der Thatsache einer vor der luetischen Infection aufgetretenen Sehstörung die richtige Diagnosestellung ermöglichen. Auf die Bedeutung, welche eine plötzlich anftretende Sehnervenaffection, die weder durch Lues, noch durch eine Intoxication bedingt ist, auch wenn sie kurz vorübergehend ist, für die Möglichkeit einer später sich entwickelnden multiplen Sklerose in prognostischer Hinsicht besitzt, ist bereits oben hingewiesen worden. Was den Verlauf, die Intensität und die klinischen Eigenthümlichkeiten der Sehstörung anbetrifft, wenn sie als Initialsymptom der Krankheit auftritt, so haben wir in unseren Fällen keine Abweichung von den Formen constatiren können, in denen sie auch sonst bei der multiplen Sklerose in die Erscheinung tritt und die durch die

1) Zur multiplen Herdsklerose. Deutsche Zeitschrift f. Nervenheilkunde. 1898. 5 u. 6. 
Beobachtungen von Charcot, Parinaud, Gnauck, Oppenheim, Uhthoff u. A. hinlänglich festgestellt sind. Auch in Bezug auf die Aetiologie haben wir keine besonderen Merkmale aufdecken können. In einem Falle wurde Ueberanstrengnng der Augen als Grund der Sehstörung angegeben, in einem anderen trat sie unmittelbar nach einem Wochenbett auf, in den meisten Fällen behaupteten die Patienten, sie wäre plötzlich, ohne jeglichen Grund und ohne vorausgegangene Erkrankung aufgetreten. Bietet uns also weder das klinische Verhalten noch die Aetiologie irgend welche Anhaltspunkte dafür, die Sehstörung für etwas mehr als ein den anderen Symptomen der Krankheit gleichwerthiges Symptom anzusehen, so scheint doch das typische Wiederkehren der Erscheinung, dass die multiple Sklerose sich in Anschlnss an eine Affection des Sehnerven anschliesst, auf die Möglichkeit eines genetischen Zusammenhanges hinzuweisen. Oppenheim ${ }^{1}$ ) hat in einer seiner Arbeiten die Vermuthung ausgesprochen, es könnte in manchen Fällen ein Krankheitserreger durch Vermittlung der Aussenhäute des Auges auf den Opticus übergreifen und von hier sich über das Centralnervensystem verbreiten. In unseren Fällen finden wir zwar keine bestimmten Angaben bezüglich einer vorausgegangenen Infection, doch muss bemerkt werden, wie leicht eine leichte Infection von den Patienten übersehen, oder die Erinnerung an dieselbe aus dem Gedächtniss verschwinden kann, wenn schon die Thatsache der Sehstörung selbst so leicht vergessen wird. ${ }^{2}$ )

Zum Schluss sei es mir gestattet, meinem verehrten Lehrer, Herrn Prof. Op penh eim, für die Anweisung zu dieser Arbeit und Ueberlassung des Materials zu der vorliegenden Mittheilung meinen Dank auszusprechen.

1) 1. c. 1896 .

2) Nach Abfassung dieser Arbeit wurde wieder ein typischer und besonders interessanter Fall dieser Art in der Oppenheim'schen Poliklinik beobachtet: B., 40jähriger Kupferschmied (Vater und Grossvater ebenfalls Kupferschmiede) war angeblich gesund bis vor 8 Jahren, damals erkrankte er mit Schwäche in den Beinen, zu der sich in der Folge Tremor, Sprachverlagsamung etc. gesellte. Status: Vorgeschrittene multiple Sklerose, nämlich spastische Paraparese, Intentionstremor, scandirende Sprache, Nystagmus, doppelseitige Blicklähmung, partielle Atrophie des linken Opticus etc. Diese ist vor $18 \mathrm{Jahren}$ in acuter Weise entstanden und hatte sich durch eine 3 Monate währende Erblindung geäussert. 